\title{
Trotz Stoma aktiv im Leben stehen
}

\section{Sicher und diskret}

" Menschen mit einem Stoma wollen eine sichere aber vor allem diskrete Versorgung, die es ihnen ermöglicht selbstbestimmt und aktiv am Leben teilzunehmen. Bereits seit 2014 bietet Coloplast mit seiner SenSura ${ }^{\circledR}$ Mio Serie hochwertige Stomaprodukte an. Seit dem 1. Juni 2017 ergänzen die 2-teiligen Ileo-Tagdrainagen das Sortiment. Mit ihrem hohen Fassungsver-

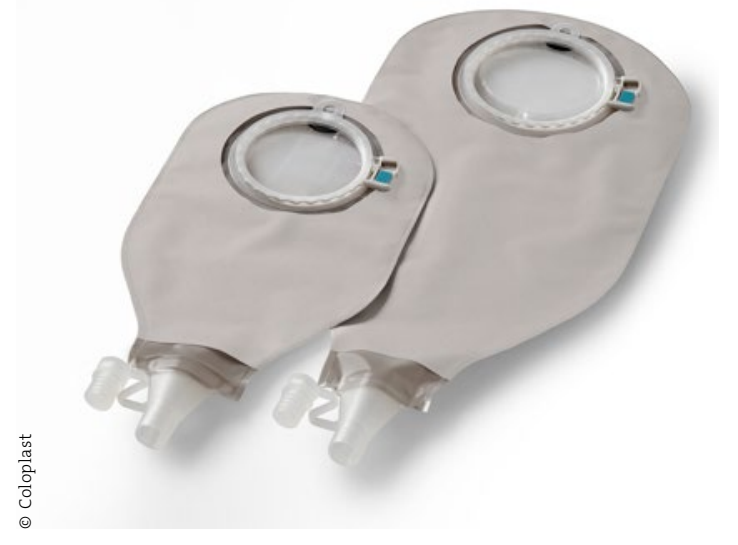

mögen eignen sie sich insbesondere für den postoperativen Einsatz, für $\mathrm{Pa}$ tienten mit Kurzdarmsyndrom sowie für die Versorgung von Ileostomiepatienten mit hohen Ausscheidungsmengen. Die Ileo-Tagdrainagen fassen über $1.000 \mathrm{ml}$ und sorgen so für möglichst lange Intervalle zwischen den Versorgungswechseln - und damit für längere Ruhezeit der Patienten. Erhältlich sind die zweiteiligen Systeme sowohl mit ClickRastringkopplung als auch mit der Flex-Klebekopplung.

Sicherheit und Komfort: Die BodyFitTechnologie ${ }^{\circledR}$ sorgt bei den neuen Produkten für eine sichere und komfortable Passform bei jeder individuellen Körperform. Der elastische Hautschutz folgt den natürlichen Körperbewegungen, dehnt sich mit der Haut und nimmt stets seine ursprüngliche Form wieder an. Die Basisplatten sind in plan, konvex light (6 mm) sowie bei der Variante mit Click-Rastringkopplung auch in konvex (9 $\mathrm{mm}$ ) erhältlich. Die Beutel verfügen über den bewährten SenSura Mio Ringfilter und eine Rückflusssperre. Bei den transparenten Beutelvarianten ist eine Messskala aufgedruckt, die Varianten mit dem diskreten, lichtgrauen Textilstoff verfügen alle über ein Sichtfenster. Bei den Auslässen kann zwischen einem Post-OP-Auslass und einem großlumigen Auslass gewählt werden. Beide Auslässe sorgen durch ihre praktische Trichterform für eine einfache Entleerung und sie lassen sich einfach und direkt an den 2.000 $\mathrm{ml}$ fassenden Coloplast-Bettbeutel anschließen.

Neu im Produktprogramm ist zudem eine diskrete Mini-Variante der SenSura Mio Ileo-Tagdrainage mit Click-Rastringverbindung. Sie wurde speziell für kleine zierliche Personen und für den häuslichen Gebrauch entwickelt und verfügt über das Volumen eines Standard-Ausstreifbeutels.

www.coloplast.de

\section{Diabetische Neuropathie}

\section{Vitamin B1-Substitution schützt Nerven und Gefäße}

") Diabetiker weisen häufig einen ausgeprägten Mangel an Vitamin B1 (Thiamin) auf. Dieses Defizit ist an der Pathogenese der diabetischen Neuropathie, einer Folgeerkrankung des Diabetes mellitus, beteiligt. Im Vitamin B1-Mangelzustand akkumulieren schädliche Verbindungen des Glukosestoffwechsels und entfalten pathogene Wirkungen an den Gefäßen. Zudem verstärkt Vitamin B1- bzw. ThiaminMangel die Auswirkungen von oxidativem Stress und fördert entzündliche Reaktionen. Folgeerkrankungen wie Neuro-, Retino- und Nephropathie sind dann möglich, erklärte Prof. Dr. Karlheinz Reiners, Hermann-Josef-Krankenhaus Erkelenz.

Im peripheren Nervensystem begünstigt Vitamin B1-Mangel die Entwicklung einer Polyneuropathie. Im
Zentralnervensystem kann der Mangel zu einer toxischen Mittelhirnschädigung wie der Wernicke-Enzephalopathie oder zu einer Neurotransmitterstörung wie der Korsakow-Psychose führen. Kompensiert werden kann dies durch die Substitution mit hoch bioverfügbarem Thiamin, erklärte Reiners. Die fettlösliche Thiamin-Vorstufe Benfotiamin (milgamma ${ }^{\circledR}$ protekt) erzielt signifikant höhere Konzentrationen an aktivem Thiamin-Diphosphat (TDP) im Plasma und in den Zellen als wasserlösliche Thiamin-Derivate. Bei der Behandlung der diabetischen Neuropathie ist das kausal wirksame Benfotiamin neben der normnahen Blutzuckereinstellung ein effektiver Therapieansatz. Eine symptomatische Therapie mit Analgetika sei dagegen aufgrund ihrer Nebenwirkungen auf
Dauer keine gute Lösung, so Reiners. Durch den frühzeitigen Ausgleich des Vitamin B1-Defizits durch Benfotiamin werden pathogene Prozesse im diabetischen Stoffwechsel kompensiert. Bei regelmäßiger Einnahme schützt es Nerven und Gefäße vor schädlichen Abbauprodukten des Glukosestoffwechsels und kann Neuropathie-Symptome wie Schmerzen, Kribbeln, Brennen oder Taubheit in den Füßen lindern. Dass Benfotiamin diese Symptome deutlich lindern kann, konnte durch Studien belegt werden.

(djb)

Pressekonferenz „Diabetes! Hören Sie auf Ihre Füße? Diabetische Neuropathie im Fokus - Aktuelles aus Wissenschaft und Praxis"; Hamburg, 24. Mai 2017 (Veranstalter: Wörwag Pharma) 